\title{
Analyzing the possibility of SARS-CoV-2 vertical transmission
}

\author{
Authors: Anastasia Kalantarova ${ }^{1}$, Nicole Josephine Zembol ${ }^{1}$, Joanna Kufel-Grabowska ${ }^{1,2}$ (mentor) \\ ${ }^{1}$ Faculty of Medicine, Poznan University of Medical Sciences, Poland \\ ${ }^{2}$ Department of Electroradiology, Greater Poland Cancer Center, Poland \\ DOI: https://doi.org/10.26800/LV-142-supp5-49

\section{Introduction:}

The novel coronavirus, termed SARS-CoV-2, and the potentially life-threatening respiratory disease associated with it, COVID-19, has rapidly evolved into a pandemic crisis. Limited information is available on the possibility of vertical transmission of SARS-CoV- 2 .

\section{Aim of the study:}

Summarize the clinical data on vertical transmission of SARS-CoV-2 and fetal outcomes.

\section{Materials and Methods:}

Electronic searches of PubMed/Medline were completed from inception to November 19, 2020. "SARS-Cov-2", "COVID-19" and "Coronavirus" terms were cross-referenced with "intrauterine transmission", "perinatal transmission", "vertical transmission of infectious disease", as well as "infant", "newborn" and "neonate". Inclusion criteria were pregnant women with a confirmed diagnosis of SARS-CoV-2 infection. Case reports written in English and published between November 17, 2019, and November 19, 2020, were screened. Data on intrauterine transmission and fetal outcomes were extracted and analyzed.

\section{Results:}

Twenty-eight articles reporting outcomes of 42 pregnant women and their infants. All women tested positive for SARS-CoV-2 either in their third $(95 \%)$ or second $(5 \%)$ trimester of pregnancy. Only 15 neonates tested positive for SARS-CoV-2. The majority of infants were delivered by C-section $(83 \%)$. Sixteen studies suggested the possibility of vertical transmission either in utero, during delivery, or breastfeeding. Various materials and methods were used to prove transmission. However, SARSCoV-2 RT-PCR testing of amniotic fluid (41\%), placenta (38\%), cord blood $(34 \%)$ were commonly implemented. The majority of newborns were isolated from the mothers, and breastfeeding was not allowed until SARS-CoV-2 RT-PCR of the mother and infant were negative. Only 1 maternal death and 1 neonatal death were reported.

\section{Conclusion:}

Vertical transmission of SARS-CoV-2 can not be ruled. It remains unclear whether neonates born to mothers positive for SARS-CoV-2 were infected in utero, intrapartum, or postpartum. Multicentre, cohort studies are needed to elucidate the role of SARS-CoV-2 during pregnancy better.

Keywords: SARS-CoV-2, pregnancy, vertical transmission 